\title{
Parametric pumping at finite frequency
}

\author{
Baigeng Wang, ${ }^{1}$ Jian Wang, ${ }^{1}$ and Hong Guo ${ }^{2}$ \\ ${ }^{1}$ Department of Physics, The University of Hong Kong, Pokfulam Road, Hong Kong, China \\ ${ }^{2}$ Center for the Physics of Materials and Department of Physics, McGill University, Montreal, Québec, Canada H3A 2T8
}

(Received 26 October 2001; published 25 January 2002)

\begin{abstract}
We report a first-principles theory for analyzing the parametric electron pump at finite frequency. The pump is controlled by two pumping parameters with phase difference $\phi$. In the zero-frequency limit, our theory recovers the well-known adiabatic result that the pumped current $I(\phi) \sim \sin \phi$. At a finite frequency, it predicts $I(\phi=0) \neq 0$ while $I(\phi=\pi) \approx 0$, consistent with recent experimental data. We discuss a possible mechanism behind the nonzero pumped current at $\phi=0$ from photon-assisted processes.
\end{abstract}

DOI: 10.1103/PhysRevB.65.073306

PACS number(s): 73.23.Ad, 72.10.Bg, 73.40.Gk

Parametric electron pump $^{1-8}$ is an interesting device, which delivers a finite dc current to the outside world at zero bias potential by cyclic variations of two device-control parameters. Recently, an adiabatic quantum electron pump was reported in an open quantum dot where the pumping signal was produced in response to the cyclic deformation of the confining potential. ${ }^{2}$ It was found that the pumping signal, $V_{d o t}(\phi)$, is sinusoidal in the phase difference $\phi$ between the two deforming potentials in the weak-pumping regime, and it becomes non-sinusoidal in the strong-pumping regime. The standard deviation of the pumping signal from its averaged value over different configurations increases linearly with the frequency of the deformation. Most notable, however, was the data ${ }^{2}$ showing $V_{d o t}(0) \neq 0$ significantly at strong pumping, whereas $V_{d o t}(\pi) \approx 0$ for all pumping strengths. Even in the weaker pumping regime, small deviations from $V_{d o t}(0)$ $=0$ could already be seen. ${ }^{2}$ The traditional and successful parametric pumping theory, ${ }^{3,4}$ valid in the adiabatic regime and up to first order in frequency, requires two pumping parameters that traverse a closed path in parameter space in each cyclic period, and the pumping signal should be proportional to the area enclosed by the path. Accordingly, if the two pumping parameters are in phase so that the enclosed area is zero, the pumping signal $V_{d o t}(0)$ should vanish. It is extremely puzzling that the experimental data ${ }^{2}$ consistently showed $V_{d o t}(0) \neq 0$, a fact that has not been understood so far, and, clearly, it calls for the development of a firstprinciples theory going beyond the adiabatic regime and low frequencies.

It is the purpose of this paper to present a theory for parametric pumping, which is valid at finite frequency. Using this theory we investigate the frequency dependence of the pumping current $I_{p}(\phi)=G_{d o t} V_{d o t}(\phi), G_{d o t}$ is a constant, and it allows us to understand why it is possible to have a pumping signal even when the pumping forces are exactly in-phase. When the frequency is low, our theory recovers the traditional adiabatic theory. ${ }^{3,4}$ As the frequency increases, we predict a nonzero $I_{p}(0)$, which is a consequence of photonassisted processes, and our theory also predicts $I_{p}(\pi) \approx 0$. These results allows us to reach the conclusion that the experimental data ${ }^{2}$ showing $V_{d o t}(0) \neq 0$ and $V_{d o t}(\pi) \approx 0$ are generic nonlinear transport features of parametric pumps at finite frequency. Furthermore, our theory predicts that at very large frequencies, $I_{p}(\pi)$ should start to deviate from zero; and most intriguing is the natural theoretical outcome that only one periodic deforming potential can produce a pumping signal at finite frequency. The latter is due to the fact that a finite frequency provides extra degrees of freedom through photon-assisted processes that is capable of playing the role of a second pumping parameter. These new predictions should be testable experimentally.

We start by considering a parametric pump that consists of a coherent quantum-scattering region attached to two ideal leads $L, R$. The leads maintain identical electrochemical potential, i.e., $\mu_{L}=\mu_{R}=\mu$. The Hamiltonian of this system is ${ }^{9}$

$$
\begin{aligned}
H_{0}= & \sum_{k, \alpha=L, R} \epsilon_{k} C_{k \alpha}^{\dagger} C_{k \alpha}+\sum_{i}\left[\epsilon_{i}(t) d_{i}^{\dagger} d_{i}+\left(t_{i} d_{i}^{\dagger} d_{i+1}+\text { c.c. }\right)\right] \\
& +\sum_{k, \alpha}\left(T_{k, \alpha} C_{k \alpha}^{\dagger} d_{j}+\text { c.c. }\right),
\end{aligned}
$$

where $C_{k \alpha}$ and $d_{i}$ are annihilation operators of electrons for the $\alpha$ lead and the scattering region at site $i$, respectively. ${ }^{10}$ The three terms describe the leads, the scattering region, and the coupling between the leads and the scattering region with the hopping matrix $T_{k, \alpha}$. In the last term, $j=1 / N$ for $\alpha$ $=L / R$. The parametric pump works by cyclic deformations of potential at two different pumping sites $i$ and $j$ in the scattering region, $V_{i / j}(t)=V_{i / j} \cos \left(\omega t+\varphi_{i / j}\right)$, where $\varphi_{i / j}$ is the phase of the pumping force.

Neglecting interaction between electrons in the ideal leads, the Keldysh nonequilibrium Green's function theory gives the following standard expression for the timedependent current $^{11}(\hbar=1)$ :

$$
\begin{aligned}
I_{L}(t)= & -q \int_{-\infty}^{t} d t_{1}\left[G_{11}^{r}\left(t, t_{1}\right) \Sigma_{L}^{<}\left(t_{1}, t\right)\right. \\
& \left.+G_{11}^{<}\left(t, t_{1}\right) \Sigma_{L}^{a}\left(t_{1}, t\right)+\text { c.c. }\right],
\end{aligned}
$$

where the Green's functions $G^{r, a,<}$ and the self-energy $\Sigma_{\alpha}$ are defined in the usual manner. ${ }^{12}$

It is tedious but straightforward to evaluate the Green's functions by iterating the equation of motion, ${ }^{11,12}$ and for our purpose it is adequate to calculate them to second order in the pumping potential $V_{i / j}(t)$, which gives the average pumped current to the second order. We note that the next 
higher nonvanishing order is the fourth. After all the Green's functions are obtained, the average pumped current is calculated from Eq. (2) by integrating time over one pumping cycle. We obtain

$$
I_{p} \equiv\left\langle I_{L}\right\rangle=I_{i i}+I_{j j}+I_{i j}+I_{j i}
$$

where

$$
\begin{aligned}
I_{i j}= & -\frac{i q V_{i} V_{j}}{1 / 4} \int \frac{d E}{2 \pi} \Gamma_{L} G_{1 i}^{0 r} G_{j 1}^{0 a}\left\{\left(f-f_{-}\right) e^{i \phi}\left[G_{i j}^{0 r-}-G_{i j}^{0 a-}\right]\right. \\
& \left.+\left(f-f_{+}\right) e^{-i \phi}\left[G_{i j}^{0 r+}-G_{i j}^{0 a+}\right]\right\}
\end{aligned}
$$

where $f \equiv f(E)$ and $f_{ \pm} \equiv f(E \pm \omega)$ are the Fermi distribution functions, $\Gamma_{L}=-2 \operatorname{Im}\left(\Sigma_{L}^{r}\right)$ is the linewidth function, $\phi \equiv \varphi_{j}$ $-\varphi_{i}$ is the phase difference between the two pumping forces. Finally, $G^{0 r} \equiv G^{0 r}(E)$ and $G^{0 r \pm} \equiv G^{0 r}(E \pm \omega)$ are the retarded Green's functions when there is no pumping force. In Eq. (4), $I_{i i}$ is obtained by setting $j=i$. Equations (3) and (4) are the main result of this work.

Before we discuss Eqs. (3) and (4) in connection with the experimental data of Ref. 2, let us first examine the lowfrequency limit of these results and show that the conventional parametric pumping theory is recovered. ${ }^{3,4}$ We expand Eq. (4) in powers of $\omega$ and only keep the linear term, this gives the adiabatic current. ${ }^{3,4}$ Note that $I_{i i}$ and $I_{j j}$ are of second order in frequency, Eq. (4) reduces to

$$
\begin{aligned}
I_{p}= & \frac{i q V_{i} V_{j} \omega}{1 / 2} \int \frac{d E}{2 \pi} \partial_{E} f \sin (\phi) \Gamma_{L} \\
& \times\left[\frac{\partial G_{11}^{0 r}}{\partial V_{i}} \Gamma_{L} \frac{\partial G_{11}^{0 a}}{\partial V_{j}}+\frac{\partial G_{1 N}^{0 r}}{\partial V_{i}} \Gamma_{R} \frac{\partial G_{N 1}^{0 a}}{\partial V_{j}}\right]+\text { c.c. },
\end{aligned}
$$

where we have used the relation $G^{r}-G^{a}=-i G^{r} \Gamma G^{a}$ and $G_{\alpha i}^{r} G_{i \beta}^{r}=\partial G_{\alpha \beta}^{r} / \partial V_{i} \cdot{ }^{13}$ Using the Fisher-Lee relation ${ }^{14,12} S$ $=-I+i \Gamma^{1 / 2} G^{r} \Gamma^{1 / 2}$, Eq. (5) reduces to exactly the same expression as that obtained from the scattering-matrix theory. ${ }^{3}$ Due to the factor of $\sin (\phi)$ in the low-frequency result (5), one obtains the familiar outcome ${ }^{3}$ that $I_{p}(\phi)=0$ at both $\phi$ $=0$ and $\phi=\pi$.

Clearly, the low-frequency result (5) does not explain the experimental result ${ }^{2}$ of $I_{p}(\phi=0) \neq 0$; we need to investigate the full result, Eq. (4), at a finite frequency. The first term on the right-hand side of Eq. (4) has a clear physical meaning: it represents the photon-absorption process indicated by the factor $\exp (i \omega t+i \phi)$. Similarly, the second term corresponds to the photon-emission process with factor $\exp (-i \omega t-i \phi)$. These two competing processes tend to cancel each other in the expression of pumped current. These photon-assisted processes are essential to understand the experimental finding ${ }^{2}$ that the pumped signal is nonzero at $\phi=0$. To see this clearly, we rewrite Eq. (3) into the following form:

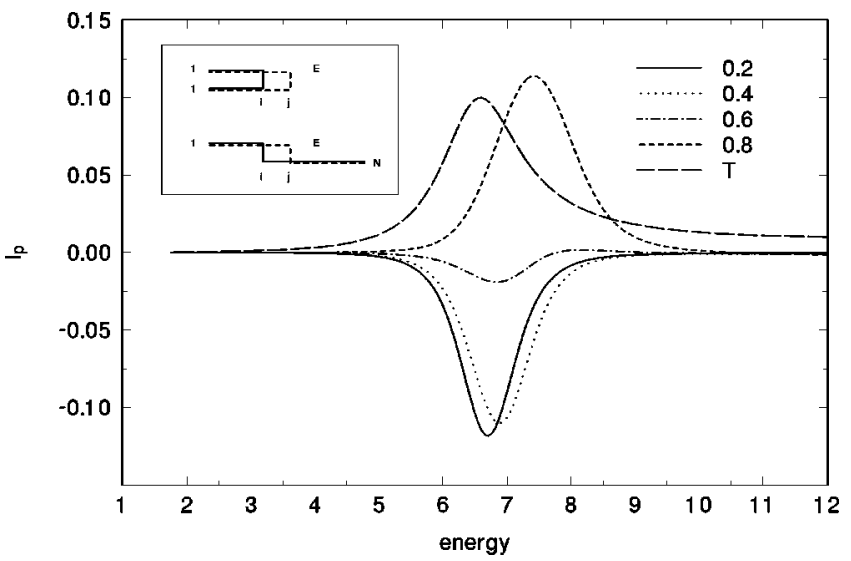

FIG. 1. Pumped current (solid line) and transmission coefficient $T$ (long dashed line) vs energy with $\phi=\pi / 2$ at $\omega=0.2,0.4,0.6,0.8$. For illustrating purpose, we shifted $T$ by multiplying a factor 0.1 . Inset: Feynman diagrams corresponds to Eq. (7). We have set $x_{i}$ $=0.02 a, x_{j}=0.25 a, V_{0}=79.2, V_{i}=V_{j}=1$.

$$
\begin{aligned}
I_{p}= & -\frac{q}{1 / 4} \int \frac{d E}{2 \pi}\left\{( f - f _ { - } ) \left[\mid g_{i}^{1}(E, E-\omega)+e^{-i \phi} g_{j}^{1}(E, E\right.\right. \\
& \left.-\omega)\left.\right|^{2}+\left|g_{i}^{N}(E, E-\omega)+e^{-i \phi} g_{j}^{N}(E, E-\omega)\right|^{2}\right]+\left(f-f_{+}\right) \\
& \times\left[\left|g_{i}^{1}(E, E+\omega)+e^{i \phi} g_{j}^{1}(E, E+\omega)\right|^{2}+\mid g_{i}^{N}(E, E+\omega)\right. \\
& \left.\left.+\left.e^{i \phi} g_{j}^{N}(E, E+\omega)\right|^{2}\right]\right\},
\end{aligned}
$$

where $g_{i}^{\alpha}(E, E-\omega) \equiv \sqrt{\Gamma_{1} \Gamma_{\alpha}}\left[G_{1 i}^{0 r}(E)\right] V_{i}\left[G_{i \alpha}^{0 r}(E-\omega)\right]$ with $\alpha=1, N$ indicating the left and right positions where the scattering region is contacted by the leads, so that $\Gamma_{1} \equiv \Gamma_{L}$ and $\Gamma_{N} \equiv \Gamma_{R}$. The propagator $G_{m n}^{0 r}$ describes the free motion of an electron from position $n$ to position $m$ in the device. Therefore, the quantity $g_{i}^{1}(E, E-\omega)$ describes the following process: a charge carrier with energy $E-\omega$ enters the device from the left lead, it absorbs a photon with frequency $\omega$ at site $i$, and then exits from the left lead with energy $E$. This process is represented by the Feynman diagram in the inset of Fig. 1. Similarly, $g_{j}^{1}(E, E-\omega)$ describes exactly the same process except that the electron absorbs a photon at position $j$. Now the physics is transparent: the first term in Eq. (6) represents an interference of the photon-absorption processes happening at positions $i, j$, i.e., the interference of the two processes in the Feynman diagram of Fig. 1. Therefore, when $\phi=0$, we have a constructive interference so that the $g_{i}^{1}(E, E-\omega)$ and the $g_{j}^{1}(E, E-\omega)$ processes add up [the factor $\exp (-i \phi)=+1]$. When $\phi=\pi$, there is a destructive interference in which the two photon-absorption processes cancel to a large extent [the factor $\exp (-i \phi)=-1]$. Exactly the same can be said for the three other terms of Eq. (6): the term involving $g_{i}^{N}(E, E-\omega)$ describes a photon-absorption process with electrons entering the device at the right lead and exiting from the left, and the terms involving $g_{i}^{1}(E, E+\omega)$ and $g_{i}^{N}(E, E+\omega)$ describe photon-emission processes.

In addition to the interference effects, the pumped current is also affected by a competition between photon emission and absorption, marked by the $\left(f-f_{-}\right)$term for absorption and the $\left(f-f_{+}\right)$term for emission, in Eq. (4). The combined 


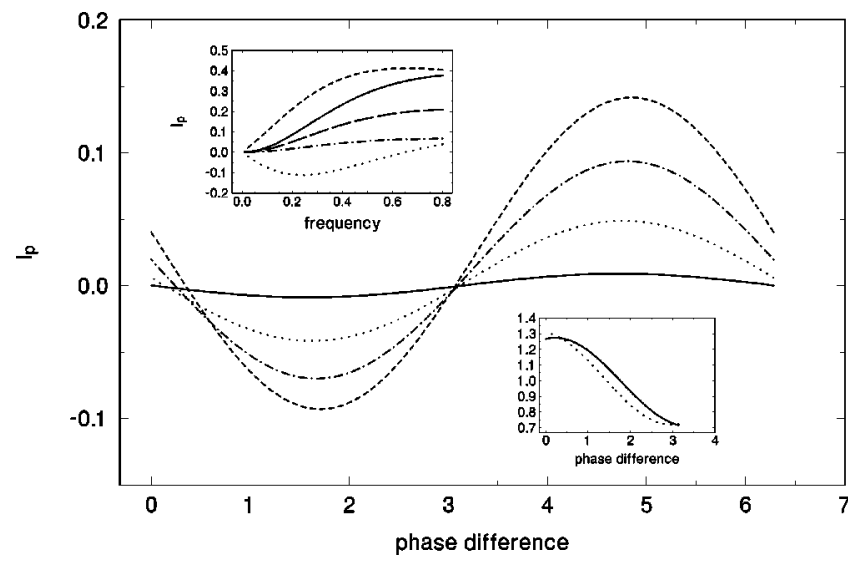

FIG. 2. $I_{p}$ vs $\phi$ for different $\omega$ at resonant point. Solid line, $\omega$ $=0.01$; dotted line, $\omega=0.05$; dash-dotted line, $\omega=0.09$; dashed line, $\omega=0.13$. Left inset: $I_{p}$ vs $\omega$ for different values of $\phi$ at resonant point. Solid line, $\phi=0$; dotted line, $\phi=\pi / 2$; dash-dotted line, $\phi=\pi$; dashed line, $\phi=3 \pi / 2$. The long dashed line is $I_{p}$ vs $\omega$ for single pumping parameter at position $x=0.2 a$. Right inset: the magnitude of pumped current at resonant point due to contributions of the first two terms (solid line) and due to the last two terms (dotted line) in Eq. (7) for $\omega=0.13$. Values $x_{i}, x_{j}, V_{0}, V_{i}$, and $V_{j}$ are as in Fig. 1.

effect, competition plus interference, can be clearly seen by expanding Eq. (6) to order $O\left(\omega^{2}\right)$, which produces a complex expression for general $\phi$, but if we concentrate only on $\phi=0$ or $\pi$, the result is much simpler and physically transparent,

$$
\begin{aligned}
I_{p}= & \frac{q}{1 / 8 \pi} \omega^{2}\left(\partial_{E_{1}}-\partial_{E_{2}}\right)\left[\left|g_{i}^{1}\left(E_{1}, E_{2}\right)+e^{i \phi} g_{j}^{1}\left(E_{1}, E_{2}\right)\right|^{2}\right. \\
& \left.+\left|g_{i}^{N}\left(E_{1}, E_{2}\right)+e^{i \phi} g_{j}^{N}\left(E_{1}, E_{2}\right)\right|^{2}\right]
\end{aligned}
$$

where one sets $E_{1}=E_{2}=E$ after taking the derivative and after the temperature is set to zero. Hence, at $\phi=0$ the constructive interference gives larger current than at $\phi=\pi$ (see also the right inset of Fig. 2 described below). The behavior of the pumped current for general $\phi$ is the result of the interplay between the photon-assisted processes and interference processes.

The above physical picture allows us to unambiguously conclude that the behavior of pumped current depends on a combination of interference and competition of photonassisted processes. The competition between absorption and emission sets an overall magnitude for the pumped current at each $\phi$. Destructive interference occurs at $\phi=\pi$, therefore $I_{p}(\pi) \approx 0$; constructive interference occurs at $\phi=0$, giving rise to a nonvanishing $I_{p}(0)$. To demonstrate this, in the right inset of Fig. 2, we plot the magnitude of pumped current versus $\phi$ at a finite frequency due to the contributions of the photon-absorption [solid line, first two terms of Eq. (6)] and photon-emission [dotted line, the last two terms of Eq. (6)] processes, respectively. Taking into account absorption and emission processes, $I_{p}(0)$ is significantly larger than $I_{p}(\pi) .{ }^{15}$ The result in this inset is obtained from a onedimensional model, which we now discuss in more detail.
Our discussion so far is completely general on the general result Eq. (4). In the following we investigate a model in a more specific manner by applying Eq. (4) to a double-barrier quantum structure, which we model by potential $U(x)$ $=V_{0} \delta(x)+V_{0} \delta(x-a)$, where $V_{0}$ is the barrier height and $a$ is the barrier separation. ${ }^{16}$ For this system the Green's function $G\left(x, x^{\prime}\right)$ can be calculated exactly. ${ }^{17}$ We choose the pumping force as $V(t)=V_{i} \delta\left(x-x_{i}\right) \sin (\omega t)+V_{j} \delta(x$ $\left.-x_{j}\right) \sin (\omega t+\phi)$. With this specific pump model, Eq. (4) can be evaluated numerically without difficulty. ${ }^{18}$

In Fig. 1, we plot the pumped current $I_{p}$ at zero temperature versus the Fermi energy at different frequencies with phase difference $\phi=\pi / 2$. For comparison, we also plot the transmission coefficient (long dashed line). The peak in the transmission coefficient indicates a quantum resonance mediated by the resonance state in the double barrier. Clearly, the pumped current $I_{p}$ also shows a resonance behavior, peaked at the same resonance state, and is largely suppressed away from it. As the frequency is increased, the pumped current reverses the sign and the peak is shifted slightly. The general feature of $I_{p}$ versus phase difference $\phi$ can be obtained from Eq. (4), which can be rewritten as $I_{p}=c_{1}$ $+c_{2} \sin \phi+c_{3} \cos \phi=c_{1}+c_{4} \sin \left(\phi+\phi_{0}\right)$, where $c_{i}$ 's and $\phi_{0}$ are constants. This indicates that $I_{p}$ has a sinusoidal behavior in $\phi$. The sinusoidal behavior is a direct consequence of the fact that our theory is valid up to the second order of the pumping amplitude. ${ }^{19}$

Figure 2 presents $I_{p}$ as a function of $\phi$ for several different frequencies at resonance where the transmission coefficient is unity. We notice that at very low frequency (solid line) $I_{p} \sim \sin (\phi)$. As the frequency increases, the amplitude of $I_{p}$ also increases. At the same time, the entire $I_{p}-\phi$ curve is shifted upward although leaving $I_{p}(\phi=\pi)$ largely unchanged. However, $I_{p}(\phi=0)$ is seen to increase significantly for the curve with $\omega=0.13$. As already discussed above, these features are due to interference of the photon-assisted processes and are consistent with the experimental observation. ${ }^{2}$ To make better comparison with experimental data, we calculated the dimensionless ratio $I(\phi=0) / I_{\max }$, where $I_{\max }$ is the maximum pumped current. Using our result shown in Fig. 1, this ratio is found to be $\sim 0.29$ at $\omega$ $=0.13(\omega=0.13$ correponds to $70 \mathrm{MHz}$, which is close to the experimental frequency). This value is quite close to that of the experimental data presented in Ref. 2. In order to make further quantitative comparisons, other system details including chaotic scattering should be included. The left inset of Fig. 2 shows $I_{p}$ as a function of frequency for several phase differences. We observe that at small frequencies, $I_{p}$ $\approx 0$ at $\phi=0$ and $\pi$. For $\phi=\pi / 2$ and $3 / 2 \pi, I_{p}$ have similar values. At larger frequencies, $I_{p}$ increases in different fashion. For instance, $I_{p}$ at $\phi=0$ increases slowly at small frequency and then has a linear behavior for larger frequencies. At $\phi=\pi / 2, I_{p}$ increases initially, reaches a maximum, and then decreases slowly. At $\phi=\pi, I_{p}$ is very small but nonzero and increases linearly with a much smaller slope, whereas $I_{p}$ at $\phi=3 \pi / 2$ is the largest among all the curves. This suggests that one should operate the parametric electron pump at $\phi$ $=3 \pi / 2$ to achieve the maximum pumped current. 


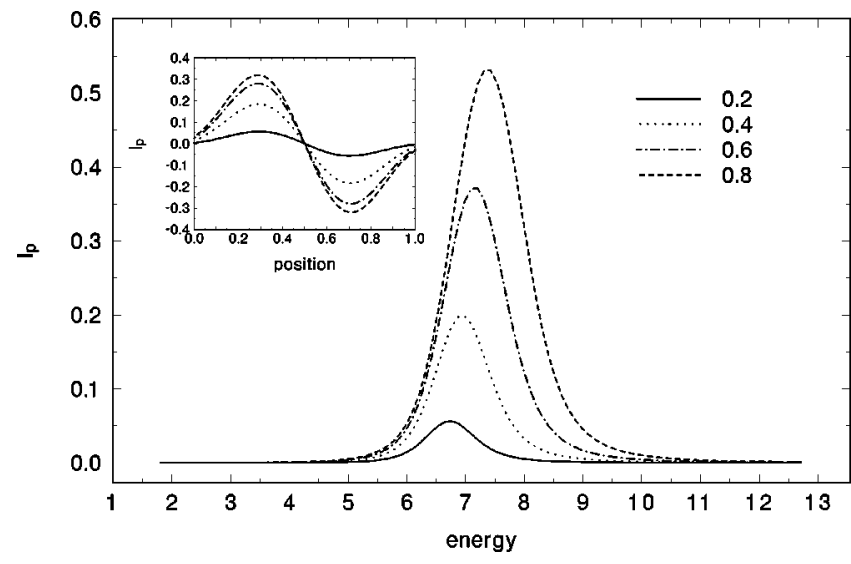

FIG. 3. $I_{p}$ vs energy at $\omega=0.2,0.4,0.6,0.8$ near resonant point with a single pumping parameter. The position of the pumping parameter is at $x=0.2 a$. Inset: $I_{p}$ vs position (in units of $a$ ) of the pumping parameter at $\omega=0.2,0.4,0.6,0.8$.

So far, we have explained the physics behind the peculiar experimental finding ${ }^{2}$ of $I_{p}(\phi=0) \neq 0$ : it is due to photonassisted processes, which are a nonlinear phenomena. However, when the two pumping forces act exactly in-phase, nothing distinguishes them (except that they act at different positions of the pump). Therefore, it is extremely interesting to ask: can one operate a pump with only one pumping parameter? To check if this is possible, we set $V_{j}=0$ in Eq. (3) and notice that the resulting $I_{p}$ still remains finite due to the first term of Eq. (3). Figure 3 plots this $I_{p}$ versus energy at different frequencies. Again, $I_{p}$ is peaked near the resonant point. As the frequency increases, the amplitude of $I_{p}$ also increases and its peak shifts towards larger energy due to the photon-assisted process. The inset of Fig. 3 shows $I_{p}$ vs the position of the single pumping site at different frequencies for a value of energy near the resonance. Due to the symmetry of the system, $I_{p}$ is antisymmetric across the center of the double barrier. It is surprising that the magnitude of the pumped current has the same order of magnitude as that pumped by two driving forces (see long dashed line in the inset of Fig. 2). The reason that a pump can operate with only one external driving force is directly related to photonassisted processes that happen at a finite frequency. It will be interesting to test this prediction by further experimental work.

In summary, we have developed a parametric pumping theory valid at finite frequency and it shows that the pumped signal for two pumping parameters in-phase can deviate from zero due to the photon-assisted process at finite frequency. This explains the anomaly at $\phi=0$ observed experimentally. This theory also suggests that even with one pumping parameter, it is possible to produce a pumped signal at finite frequency whose amplitude is of the same order of magnitude as that of two pumping parameters.

Note added. After the paper was submitted, we were made aware of a recent work by Brouwer, ${ }^{20}$ which examined magnetic-field symmetry of the pump. It suggested that rectification effect might be important in understanding the $\phi$ $=0$ anomaly.

We gratefully acknowledge support by a RGC grant from the SAR Government of Hong Kong under Grant No. HKU 7115/98P. H.G. is supported by NSERC of Canada and FCAR of Québec.
${ }^{1}$ B.L. Altshuler and L.I. Glazman, Science 283, 1864 (1999).

${ }^{2}$ M. Switkes, C. Marcus, K. Capman, and A.C. Gossard, Science 283, 1905 (1999).

${ }^{3}$ P.W. Brouwer, Phys. Rev. B 58, R10 135 (1998).

${ }^{4}$ F. Zhou, B. Spivak, and B.L. Altshuler, Phys. Rev. Lett. 82, 608 (1999).

${ }^{5}$ T.A. Shutenko, I.L. Aleiner, and B.L. Altshuler, Phys. Rev. B 61, 10366 (2000).

${ }^{6}$ I.A. Aleiner, B.L. Altshuler, and A. Kamenev, Phys. Rev. B 62, 10 373 (2000).

${ }^{7}$ M.G. Vavilov, V. Ambegaokar, and I.L. Aleiner, Phys. Rev. B 63, 195313 (2001)

${ }^{8}$ Y.D. Wei, J. Wang, H. Guo, and C. Roland, Phys. Rev. B 64, 115321 (2001); J. Wang, Y.D. Wei, B.G. Wang, and H. Guo, Appl. Phys. Lett. 79, 3977 (2001).

${ }^{9}$ We focus on developing a nonadiabatic theory, which is crucial in understanding the $\phi=0$ experimental anomaly, rather than on the chaotic nature of the quantum dot in the pump of Ref. 2 .

${ }^{10}$ Since the pumping potential in the scattering region is position dependent, it is easiest to work in a real-space tight-binding representation rather than in the momentum space. The scattering region has $N$ sites. In the numerics we choose $N=400$ to make sure the convergence of results.

${ }^{11}$ A.P. Jauho, N.S. Wingreen, and Y. Meir, Phys. Rev. B 50, 5528 (1994).

12 M.P. Anantram and S. Datta, Phys. Rev. B 51, 7632 (1995).

13 V. Gasparian, T. Christen, and M. Buttiker, Phys. Rev. A 54, 4022 (1996).

14 D.S. Fisher and P.A. Lee, Phys. Rev. B 23, 6851 (1981).

${ }^{15}$ If $x_{i}$ is closer to $x_{j}$, the interference effect will be drastically enhanced. For instance, we have $I_{p}(0) / I_{p}(\pi) \sim 130$ for $x_{i}$ $=0.13 a$ and $x_{j}=0.25 a$ as compared with $I_{p}(0) / I_{p}(\pi) \sim 5$ for $x_{i}=0.02 a$ and $x_{j}=0.25 a$ shown in the right inset of Fig. 2 .

${ }^{16}$ If the double barrier is asymmetric, the pumped current will be suppressed.

${ }^{17}$ M.K. Yip, J. Wang, and H. Guo, Z. Phys. B: Condens. Matter 104, 463 (1997).

${ }^{18}$ In the numerics we set units as $\hbar=2 m=1$. For a system fabricated by GaAs with $a=1.0 \mu \mathrm{m}$, which corresponds to the linear dimension of the quantum dot in the experiment of Ref. 2, our energy unit is $E=2.3 \mu \mathrm{eV}$.

${ }^{19}$ The strong-pumping regime has been considered in Y.D. Wei et al., Phys. Rev. B 62, 9947 (2000).

${ }^{20}$ P.W. Brouwer, Phys. Rev. B 63, 121303 (2001). 